\title{
RESSECÇÕES HEPÁTICAS COM OCLUSÃO VASCULAR AFERENTE: ANÁLISE DE FATORES DE RISCO
}

\author{
HEPATIC RESECTION WITH AFFERENT VASCULAR OCCLUSION: \\ ANALYSIS OF RISK FACTORS
}

\author{
Enilde Eloena Guerra, TCBC-RS \\ Luiz M. Pereira-Lima, TCBC-RS ${ }^{2}$
}

\begin{abstract}
RESUMO: Objetivo: O controle da perda sangüínea nas cirurgias de ressecção hepática está associado à diminuição dos índices de morbimortalidade. As técnicas para minimizar a hemorragia transoperatória são aquelas associadas à redução do fluxo sanguiíneo ao fígado, através da oclusão vascular aferente (manobra de Pringle) ou exclusão vascular total do órgão. O objetivo deste estudo foi o de avaliar uma série de hepatectomias parciais com oclusão do fluxo sangüíneo aferente, em pacientes portadores de doenças benignas e malignas. Métodos: Foram analisadas 60 hepatectomias em 59 pacientes com clampeamento do pedículo hepático quanto a possíveis fatores de risco para morbidade e mortalidade, a relação entre o tempo de isquemia hepática e a variação das transaminases, tempo de protrombina e bilirrubinas, e destes, com a evolução pós-operatória. Resultados: A prevalência de complicações pós-operatórias foi de 43,3\% e a mortalidade de $6,7 \%$. O fator de risco significativo para mortalidade foi tempo cirúrgico mais prolongado. Para a morbidade pós-operatória, os fatores de risco foram idade acima de 60 anos, cirurgia por neoplasia maligna, parênquima hepático anormal, perda sangüínea necessitando reposição de mais de uma unidade de sangue e outra cirurgia abdominal concomitante. $\mathrm{Na}$ análise multivariada por regressão logística, estes fatores de risco foram reduzidos para parênquima hepático anormal. Conclusões: $O$ tempo de isquemia não apresentou relação com a morbimortalidade pós-operatória. A variação das transaminases foi mais acentuada nos casos com maior tempo de isquemia, porém essas retornaram aos níveis pré-operatórios em aproximadamente uma semana. A variação das transaminases não foi diferente entre os pacientes com e sem morbidade pósoperatória.
\end{abstract}

Descritores: Fígado, Isquemia, Morbidade; Clampeamento.

\section{INTRODUÇÃO}

A intervenção cirurgíca hepática tem sido um grande desafio na história evolutiva da cirurgia e um sólido conhecimento da anatomia funcional do fígado é uma condição imprescindível para o desenvolvimento das hepatectomias. Este tipo de cirurgia tem sido realizado com progressiva freqüência no tratamento de tumores primários ou metastáticos e, especialmente nas últimas duas décadas, o refinamento da técnica de ressecção hepática, o desenvolvimento de rotinas específicas de anestesia, suporte ventilatório e hemodinâmico estão diretamente associados com a diminuição dos índices de morbidade e mortalidade ${ }^{1}$, quando a cirurgia é realizada em centros especializados ${ }^{2-4}$ e em pacientes rigorosamente selecionados ${ }^{5}$.

As taxas de mortalidade operatória, para casos de neoplasias malignas, foram reduzidas de, aproximadamente, $20 \%$, há 20 anos, para índices de $2 \%$ a $10 \%$ nos dias de hoje $\mathrm{j}^{5,6}$. A incidência de complicações pós-operatórias ainda permanece relativamente elevada com uma taxa que varia entre $10 \%$ e $50 \%{ }^{5,7}$ e, mesmo para tratamento de doenças benignas, encontramos índices de morbidade, defi-

1. Mestre em Medicina: Cirurgia pela UFRGS. Preceptora da Residência Médica em Cirurgia Geral no HNSC - Porto Alegre.

2. Professor Titular do Departamento de Cirurgia da FFFCMPA. Professor Adjunto do Departamento de Cirurgia da Faculdade de Medicina da UFRGS.

Recebido em 20/12/2000

Aceito para publicação em 21/08/2001

Trabalho realizado no Complexo Hospitalar Santa Casa e Hospital de Clínicas de Porto Alegre. Curso de Pós-Graduação em Medicina. Faculdade de Medicina. Universidade Federal do Rio Grande do Sul 
nidos como qualquer complicação pós-operatória, entre $10 \%$ e $21 \%{ }^{8,9}$. A grande maioria de complicações pós-operatórias maiores está relacionada com excessiva perda sangüínea no período perioperatório, insuficiência hepática ou processos infecciosos ${ }^{5}$.

A hemorragia profusa e a embolia aérea persistem como complicações graves durante ressecções de lesões hepáticas volumosas, especialmente aquelas localizadas em posição central no parênquima. $O$ controle da perda sanguínea, no transoperatório de ressecções hepáticas extensas, deve ser o objetivo primordial do profissional envolvido com este tipo de cirurgia ${ }^{10}$.

A obstrução ao fluxo sangüíneo hepático, tanto parcial, como descrito por Pringle ${ }^{11}$, quanto total, está relacionada com diminuição da perda sangüínea transoperatória, sem referência a dano ou falência hepática persistente, mesmo quando ocorrem períodos prolongados de isquemia tecidual, ou seja, acima de 60 minutos. A redução na necessidade de reposição de sangue está associada à menor morbidade e à menor incidência de complicações pósoperatórias $^{3,12}$.

O sangramento transoperatório também tem uma estreita relação com o aumento da incidência de sepse abdominal pós-hepatectomia. Na série apresentada por Yanaga et al. entre os pacientes que desenvolveram sepse de origem abdominal e/ou abscesso intra-abdominal, $68,4 \%$ deles sofreram perda sangüínea maior ou igual a $3.000 \mathrm{ml}$ durante $\mathrm{o}$ ato cirúrgico $(\mathrm{p}<0,001)$ quando comparado àqueles sem complicações infecciosas ${ }^{12}$.

O objetivo geral deste estudo visa analisar uma série de ressecções hepáticas com oclusão vascular aferente, realizadas pela mesma equipe cirúrgica, quanto aos fatores de risco para a morbidade e mortalidade perioperatória de hepatectomias; a variação das transaminases e tempo de protrombina em relação ao tempo de isquemia e a comparação do tempo de clampeamento pedicular aferente com a morbidade no período pós-operatório imediato.

\section{MÉTODOS}

Esta pesquisa se caracteriza por um estudo de coorte histórico, de caráter observacional, descritivo e analítico A amostra deste estudo foi constituída por pacientes do sexo masculino e feminino submetidos à cirurgia para ressecções hepáticas por doença benigna ou neoplasia maligna, no Hospital de Clínicas de Porto Alegre e no Complexo Hospitalar Santa Casa, por um período de cinco anos.

A avaliação pré-operatória foi realizada de forma a traçar um perfil dos pacientes quanto ao seu estado físico antes da cirurgia. Os exames laboratoriais considerados na avaliação desses pacientes foram hemograma, provas de função renal e provas de função hepática (bilirrubinas, fosfatase alcalina, transaminase glutâmico-oxalacética ou aspartato aminotransferase (AST), transaminase glutâmicopirúvica ou alanina aminotransferase (ALT) e tempo de protrombina). Os exames de imagem específicos para patologia hepática foram a ultra-sonografia e a tomografia computadorizada abdominais. De acordo com a orienta- ção do serviço, nenhum paciente foi submetido à biópsia hepática percutânea pré-operatória.

Para análise das hepatectomias foram avaliadas as seguintes variáveis : idade (dividida em três faixas etárias, até 40 anos, de 40 a 60 anos e acima de 60 anos), sexo, índice de massa corporal (IMC), bilirrubina pré-operatória, patologia de base (doença benigna ou maligna), tipo de incisão, forma e tempo de clampeamento, extensão da ressecção hepática, associação com outro tipo de cirurgia, perda sangüínea, reposição hídrica e de sangue, tempo cirúrgico, doença hepática associada e tempo de hospitalização pós-operatória. Paralelamente, foi determinada a variação das transaminases, bilirrubinas e tempo de protrombina no pós-operatório, a partir do período pré-operatório até a normalização dos valores.

Foi utilizada incisão subcostal bilateral, com ou sem prolongamento mediano, em direção ao apêndice xifóide. A critério do cirurgião, em algumas ressecções, foi necessário associação de incisão abdominal com toracotomia direita. Após a revisão da cavidade abdominal e avaliação da ressecabilidade da lesão, o fígado era completamente liberado, incluindo ramos da veia cava retrohepática e só então iniciado o período de isquemia. A técnica de impedimento vascular utilizada em todos os pacientes foi a oclusão total do pedículo hepático com a utilização de uma pinça de clampeamento vascular do tipo Satinsky sem dissecção prévia dos elementos vasculares ou da via biliar extra-hepática. Esta manobra era realizada de forma contínua ou de forma intermitente, sendo esta última realizada com intervalos sem isquemia de 5 minutos entre os diversos clampeamentos e período máximo de oclusão de 30 minutos. Foram necessários de dois até cinco períodos de impedimento do fluxo sangüíneo aferente. Nos pacientes com fígado cirrótico sempre foi utilizado o clampeamento intermitente com períodos máximos de 15 minutos de isquemia.

A partir do exame anatomopatológico da peça cirúrgica, o parênquima hepático foi estratificado em quatro categorias: normal, com esteatose, colestático ou cirrótico e, posteriormente, para análise, novamente classificado em normal e anormal.

Os pacientes, neste estudo, foram divididos em dois grupos, conforme a morbidade, sendo o grupo I composto por pacientes que apresentaram evolução pós-operatória sem intercorrências e o grupo II, por pacientes que apresentaram complicações pós-operatórias.

O dano ao parênquima hepático causado pela isquemia normotérmica foi avaliado pelas curvas de variação das transaminases, tempo de protrombina e bilirrubinas no período pré-operatório imediato e pós-operatório. O tempo de clampeamento foi dividido em quatro grupos com diferença de 30 minutos entre os períodos (até $30 \mathrm{~min}$; de 31 a $60 \mathrm{~min}$; de 61 a 90min e acima de 90min).

A análise da relação entre os dois grandes grupos divididos de acordo com a morbidade e mortalidade foi realizada, de forma univariada, através do teste não paramétrico de Mann-Whitney U e o Teste de Qui-quadrado. Quando a amostra tornou-se reduzida por critérios de estratificação, foi utilizado o teste exato de Fisher. Paralela- 
mente, foi realizada análise de regressão logística multivariada para o desfecho morbidade. A medida de efeito utilizada foi a razão de chances e os respectivos intervalos de confiança. Para análise da variação das transaminases, nos quatro períodos de isquemia, foi utilizado o Teste de Kruskal-Wallis. O intervalo de confiança utilizado para todos os testes foi de $95 \%$.

\section{RESULTADOS}

Um total de 59 pacientes foram submetidos a 60 ressecções hepáticas (recidiva de hepatocarcinoma). Vinte pacientes eram masculinos $(33,9 \%)$ e 39 femininos $(66,1 \%)$. A média de idade foi de 48,4 anos e variabilidade de 16 a 84 anos. A doença básica foi benigna em 28 casos $(46,7 \%)$ e neoplasia maligna, em 32 (53,3\%).

A presença de doença do parênquima hepático associada à afecção de base foi identificada em 10 casos: cirrose em $3(5,0 \%)$, esteatose em $5(8,3 \%)$ e colestase em 3 casos $(5,0 \%)$. Em 44 pacientes, o parênquima hepático era normal.

A "manobra de Pringle" foi realizada de forma contínua em 37 operações $(61,7 \%)$ e de forma intermitente em $23(38,3 \%)$. O tempo médio de clampeamento de forma contínua foi de 42,97 minutos, variando de 15 a 80 minutos. Nos casos de clampeamento intermitente, o intervalo máximo de isquemia foi de 30 minutos e o tempo total de oclusão vascular foi, em média, de 62,09 minutos com variabilidade de 25 a 120 minutos.

Quanto a extensão da ressecção hepática, foram realizadas 35 hepatectomias maiores, ou seja, mais de três segmentos conforme a classificação de Couinaud ${ }^{16},(58,3 \%)$ e 25 menores $(41,7 \%)$. A perda sanguínea transoperatória foi avaliada através da reposição de sangue que foi necessária em 34 pacientes $(56,7 \%)$, com uma média de 2,7 unidades e variação de uma a 10 unidades.

O tempo cirúrgico variou de 130 a 600 minutos, com uma média de $306 \pm$ 95,5 minutos.

O tempo médio de hospitalização pós-operatória foi de 14,9 dias, variando de cinco a 86 dias.

Quatro pacientes evoluíram para óbito $(6,7 \%)$, todos submetidos a hepatectomias por doença maligna. A média de idade destes pacientes era de 48,0 $\pm 15,2$ anos. Os óbitos ocorreram entre o sétimo e o vigésimo-sexto dia de evolução pós-operatória.

Dois casos foram submetidos a clampeamento de forma contínua e dois de forma intermitente, com períodos médios de isquemia de 63 minutos e 81,5 minutos, respectivamente. $\mathrm{Na}$ análise estatística, o tempo de clampeamento não foi um fator significativo para mortalidade $(\mathrm{p}=0,0748)$.

O tempo cirúrgico médio de 451,67 £ 101,04 minutos foi o único fator estatisticamente significativo nesta amostra quando comparado com os pacientes que não foram a óbito $(297,6$ minutos $\pm 89,15)(\mathrm{p}=0.0244)$.

Em 34 casos, a evolução pós-operatória foi sem intercorrências (grupo I) e em 26 (grupo II) foram identificadas complicações pós-operatórias gerais, sendo a mais freqüente infecção respiratória. As complicações cirúrgicas, diretamente relacionadas à ressecção hepática e que necessitaram de reintervenção, estiveram presentes em 5 casos $(8,3 \%)$, dos quais, quatro, para drenagem de abscesso abdominal e uma ressutura de parede para correção de evisceração.

A Tabela 1 resume os resultados dos fatores de risco para morbidade pós-operatória.

A idade foi agrupada por faixas etárias em três níveis, sendo $27,6 \%$ dos casos em pacientes com menos de 40 anos de idade, $46,6 \%$ na faixa de 40 a 59 anos e $25,9 \%$ acima de 59 anos. A ocorrência de complicações foi de $18,7 \%$ na primeira faixa, $44,5 \%$ na segunda e $66,7 \%$ no terceiro nível, com significância estatística ( $\mathrm{p}=0,02618)$.

A dosagem de bilirrubina total (BT) na avaliação préoperatória entre os dois grupos através do ponto de corte em normal (até $1,2 \mathrm{mg} / \mathrm{dl}$ ) mostrou um resíduo ajustado do qui-quadrado maior que $\pm 1,96$, o que indica uma diferença significativa entre a freqüência esperada e a observada (** Tabela 1). Pelo teste exato de Fisher, não houve significância estatística com $\mathrm{p}=0,05976$.

A média de transfusões sangüíneas foi de 1,18 $\pm 1,61$ unidades no grupo I e de $2,04 \pm 2,29$ no grupo II ( $p=0,1173$ ).

A partir desses dados, foi realizada análise multivariada e o fator de risco significativo foi parênquima hepático anormal; a indicação de cirurgia por patologia maligna, apesar de não significativa, apresentou níveis próximos a uma significância limítrofe (Tabela 2). Estes resultados podem ter sido alterados por causa do número da amostra, pois esta análise multivariada, em amostras pequenas, pode estimar um risco acima do normal.

Foram analisadas a evolução das transaminases (Figuras 1 e 2) e bilirrubina total (Figura 3) quanto à presença de complicações. Na análise estatística, a evolução da AST e ALT não foi diferente entre os dois grupos $(p>0,05)$. A evolução das bilirrubinas (BT) torna-se significativamente diferente entre o grupo que apresentou complicações pósoperatórias e o grupo sem complicações, a partir do segundo dia de pós-operatório (P.O.), com p=0,0125.

Para avaliação das transaminases (Figuras 4 e 5), bilirrubina total (Figura 6) e tempo de protrombina (TP) (Figura 7), os períodos de impedimento do fluxo sangüíneo aferente foram estratificados em quatro faixas com divisões de 30 minutos. Pode-se observar uma tendência à normalização dos valores com a evolução do tempo no pósoperatório. A diferença de AST do primeiro ao quarto dia de P.O foi estatisticamente significativa, exceto na comparação entre os grupos I e II. A diferença nas dosagens de ALT foi significativa a partir do $3^{\circ}$ P.O. quando comparados os grupos I e III, grupos I e IV e grupos II e IV ( $p<0,05)$. As curvas de evolução de bilirrubina total e tempo de protrombina não apresentaram diferenças estatisticamente significativas em relação ao tempo de clampeamento $(\mathrm{p}>0,05)$.

\section{DISCUSSÃO}

A cirurgia hepática vem se desenvolvendo em um grande número de centros especializados. Em nosso meio, a sua evolução ainda é recente, porém, vários grupos têm se dedicado ao seu aprimoramento ${ }^{2,14}$. Uma melhor apreciação das indicações das cirurgias, desenvolvimento dos 
Tabela 1

Análise dos Fatores de Risco para Morbidade

\begin{tabular}{|c|c|c|c|}
\hline Fatores de risco & $\begin{array}{c}\text { Grupo I } \\
n=34 \\
(56,7 \%)\end{array}$ & $\begin{array}{c}\text { Grupo II } \\
n=26 \\
(43,3 \%)\end{array}$ & \multirow[t]{2}{*}{$p$} \\
\hline \multicolumn{3}{|l|}{ Idade } & \\
\hline • média (DP) & $45,15 \quad(13,88)$ & $52,72 \quad 15,51)$ & $0,0563(\mathrm{NS})$ \\
\hline$\cdot \leq 60$ anos & $65,1 \%$ & $34,9 \%$ & \\
\hline$\cdot>60$ anos & $33,3 \%$ & $66,7 \%$ & 0,0323 \\
\hline \multicolumn{4}{|l|}{ Sexo } \\
\hline - masculino (\%) & $(26,5)$ & $(46,2)$ & $0,11317(\mathrm{NS})$ \\
\hline - feminino $(\%)$ & $(73,5)$ & $14 \quad(53,8)$ & \\
\hline \multicolumn{4}{|l|}{ IMC } \\
\hline • média (DP) & $24,96 \quad(4,08)$ & $25,32 \quad(4,18)$ & $0,7540(\mathrm{NS})$ \\
\hline \multicolumn{4}{|l|}{ Patologia básica } \\
\hline • benigna $(\%)$ & $20 \quad(58,8)$ & $8 \quad(30,8)$ & 0,03089 \\
\hline - maligna (\%) & $(41,2)$ & $(69,2)$ & $*$ \\
\hline \multicolumn{4}{|l|}{ Bilirrubina total pré-op } \\
\hline$\bullet 1,2 \mathrm{~g} / \mathrm{dl}$ & $100,0 \%$ & $84,2 \%$ & 0,05976 \\
\hline$\cdot>1,2 \mathrm{~g} / \mathrm{dl}$ & 0,0 & $15,8 \%$ & $* *$ \\
\hline \multicolumn{4}{|l|}{ Parênquima hepático } \\
\hline - $\operatorname{normal}(\%)$ & $(90,6)$ & $(65,2)$ & 0,03751 \\
\hline - $\operatorname{anormal}(\%)$ & $(9,4)$ & $(34,8)$ & $*$ \\
\hline \multicolumn{4}{|l|}{ Incisão cirúrgica } \\
\hline - com toracotomia $(\%)$ & $(3,0)$ & $(8,0)$ & $0,57221(\mathrm{NS})$ \\
\hline - sem toracotomia $(\%)$ & $(97,0)$ & $23 \quad(92,0)$ & \\
\hline \multicolumn{4}{|l|}{ Tipo de clampeamento } \\
\hline • contínuo (\%) & $(67,6)$ & $(53,8)$ & $0,27591(\mathrm{NS})$ \\
\hline - intermitente $(\%)$ & $(32,4)$ & $(46,2)$ & \\
\hline \multicolumn{4}{|l|}{ Tempo de clampeamento (min) } \\
\hline$\bullet$ média (DP) & $\begin{array}{c}48,21 \\
(24,08)\end{array}$ & $\begin{array}{c}53,44 \\
(26,74)\end{array}$ & $0,4333(\mathrm{NS})$ \\
\hline \multicolumn{4}{|l|}{ Extensão da ressecção } \\
\hline$\bullet$ maiores $(\%)$ & $(52,9)$ & $(65,4)$ & $0,33264(\mathrm{NS})$ \\
\hline - menores $(\%)$ & $(47,1)$ & $(34,6)$ & \\
\hline \multicolumn{4}{|l|}{ Cirurgia concomitante } \\
\hline - $\operatorname{sim}(\%)$ & $(0)$ & $(30,8)$ & 0,00061 \\
\hline • não (\%) & $(100,0)$ & $(69,2)$ & $*$ \\
\hline Reposição hídrica: crist/colóides & $4300 \quad(2702,5) /$ & $4676(1933,1) /$ & \\
\hline$\bullet$ média $(\mathrm{DP}) \mathrm{ml}$ & $847 \quad(508,3)$ & $910,7(303,9)$ & $0,2551(\mathrm{NS})$ \\
\hline \multicolumn{4}{|l|}{ Reposição de sangue } \\
\hline • até 1 unidade $(\%)$ & $(72,7)$ & $(46,1)$ & 0,03773 \\
\hline - acima de 1 unidade (\%) & $(27,3)$ & $(53,9)$ & \\
\hline $\begin{array}{c}\text { Tempo cirúrgico (minutos) } \\
\bullet \text { média (DP) }\end{array}$ & & & \\
\hline$\bullet$ média $(\mathrm{DP})$ & $290,17 \quad(95,01)$ & $(94,59)$ & $0,1469(\mathrm{NS})$ \\
\hline
\end{tabular}

Grupo I - sem complicações; Grupo II - complicações pós-operatórias

* - Estatisticamente significativo; NS - não significativo

** - Vide texto DP - desvio padrão 
Tabela 2

Razão de Chances para Fatores de Risco de Complicações Pós-operatórias

\begin{tabular}{|c|c|c|c|}
\hline Fatores de Risco & $P$ & $O . R$. & I.C. $95 \%$ \\
\hline Parênquima hepático anormal & 0,0219 & 8,33 & {$[1,36 ; 51,06]$} \\
\hline Neoplasia maligna & $0,0551(\mathrm{NS})$ & 3,95 & {$[0,97 ; 16,15](\mathrm{NS})$} \\
\hline
\end{tabular}

O.R. - razão de chances (odds ratio)

I.C. $95 \%$ - intervalo de confiança de $95 \%$

$N S$ - não significativo

meios de suporte perioperatório e o maior conhecimento da técnica cirúrgica tendem a melhorar os índices de morbidade e mortalidade.

Os resultados das ressecções hepáticas podem ser aprimorados com o uso de processos de impedimento do fluxo sangüíneo, embora ainda não seja um consenso, pois a isquemia, também, poderia ser um fator de risco para complicações nestes pacientes ${ }^{15,16}$. A seleção dos pacientes também tem sido apontada como fator prognóstico positivo $^{5}$ e essa deve estar apoiada em estudos de fatores de

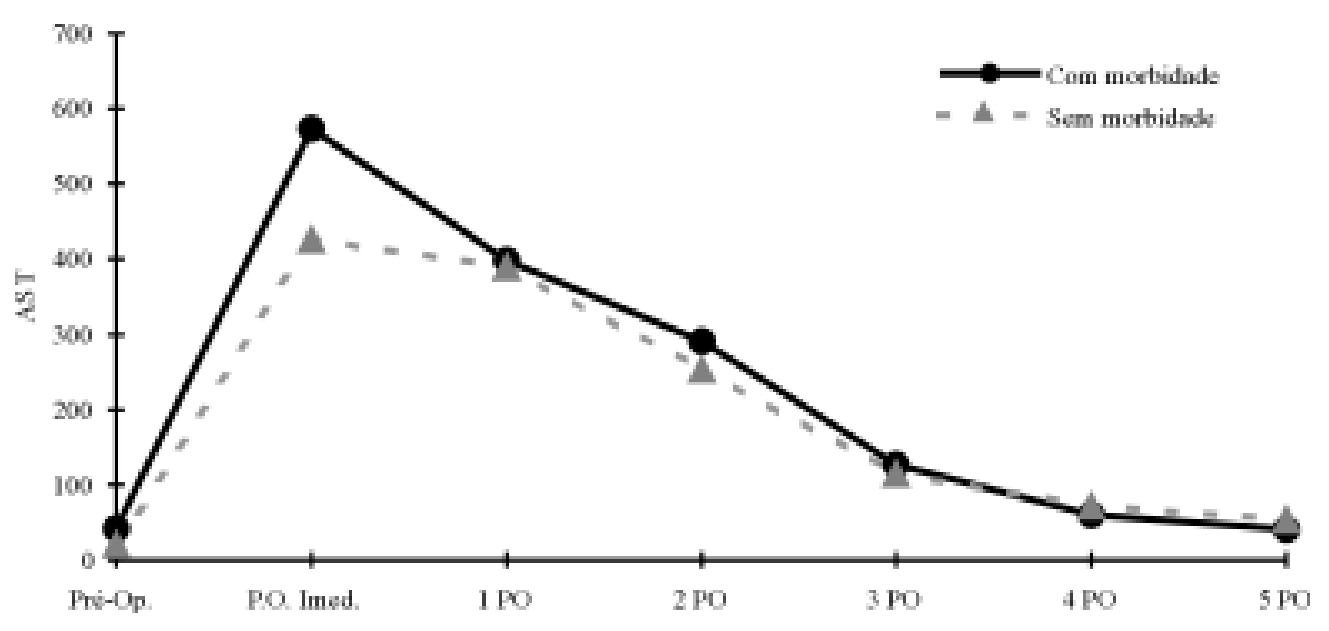

Figura 1 - Médias de transaminase AST (TGO) nos exames pré e pós-operatório, em relação à morbidade.

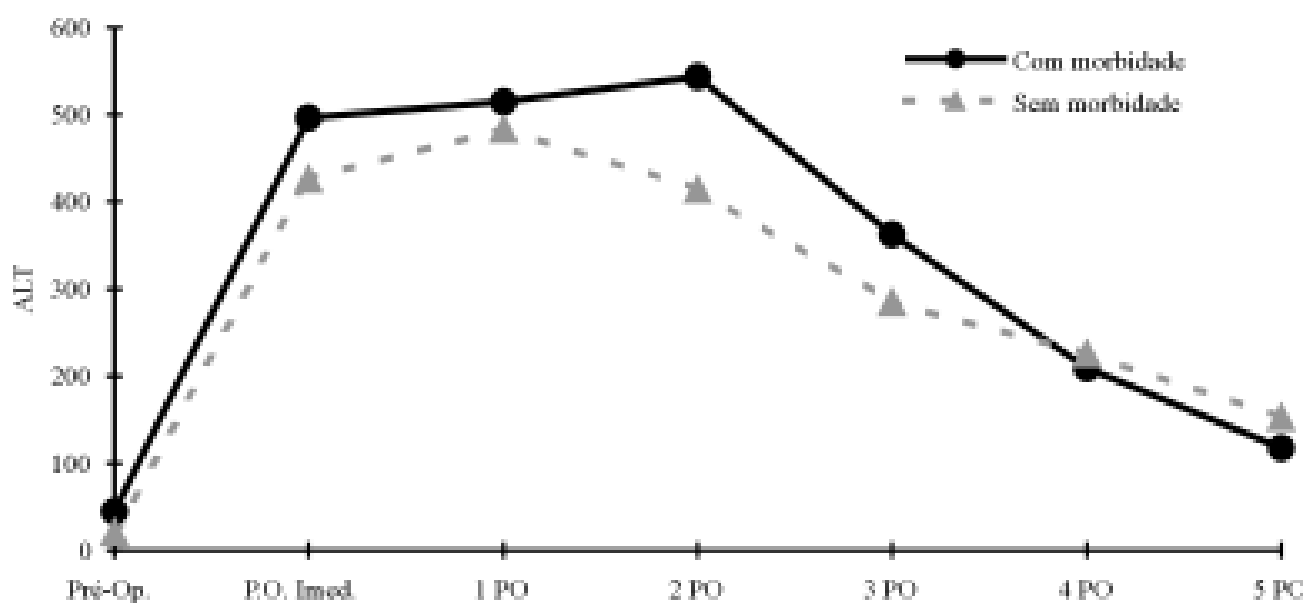

Figura 2 - Médias de transaminase ALT (TGP) nos exames pré e pós-operatório, em relação à morbidade. 


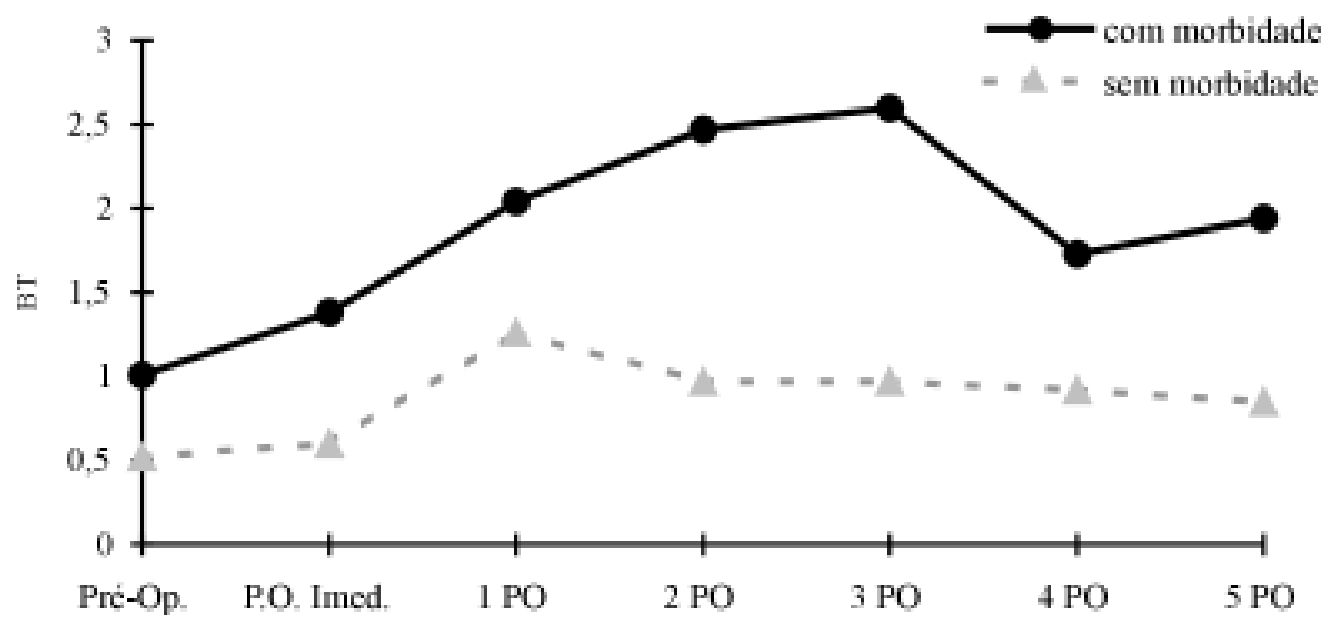

Figura 3 - Médias de bilirrubina total (BT) nos exames pré e pós-operatório, em relação à morbidade.

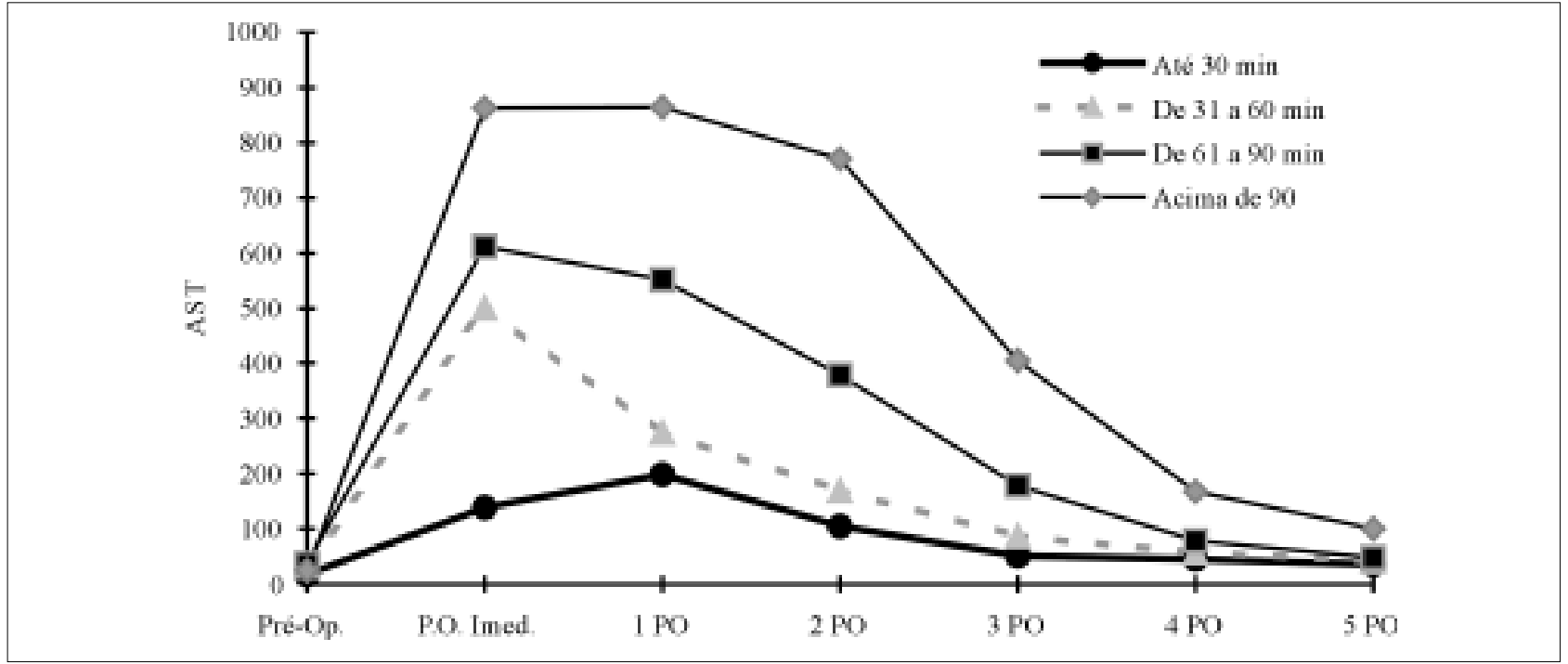

Figura 4 - Médias de transaminases AST (TGP) nos exames pré e pós-operatório, em relação ao tempo de clampeamento.

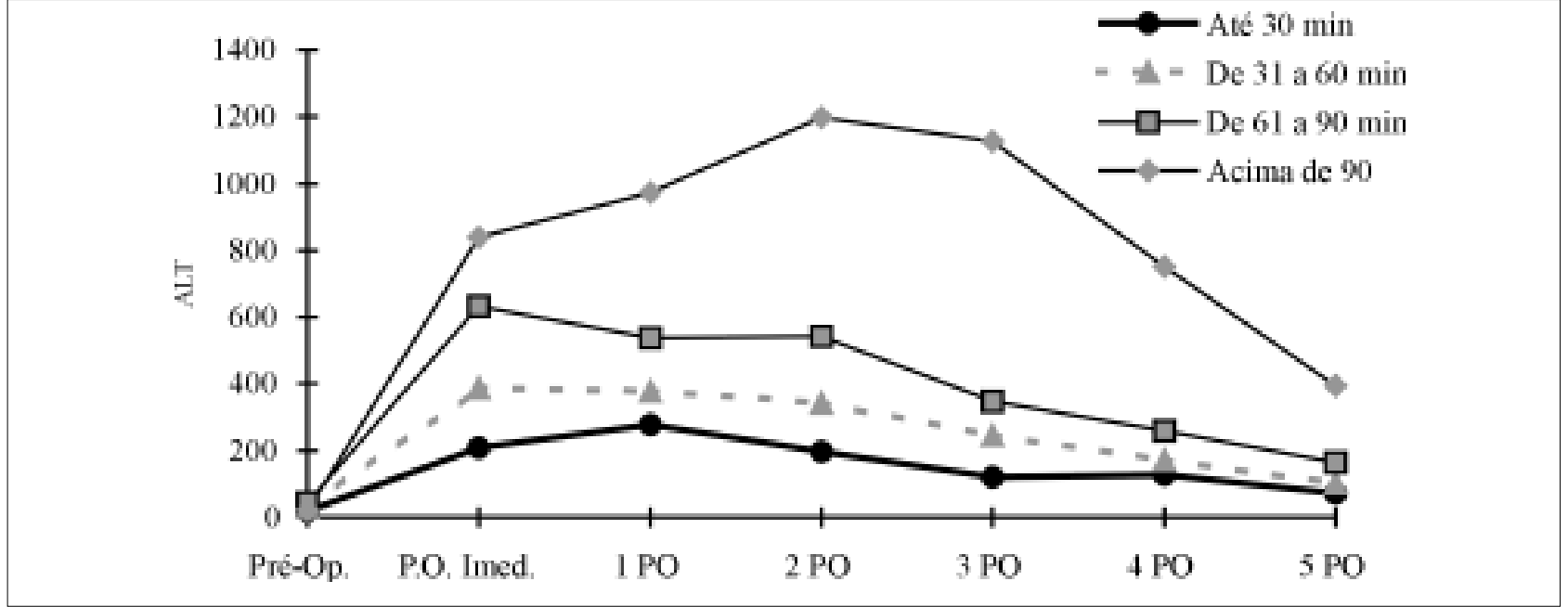

Figura 5 - Médias de transaminases $A L T(T G P)$ nos exames pré e pós-operatório, em relação ao tempo de clampeamento. 


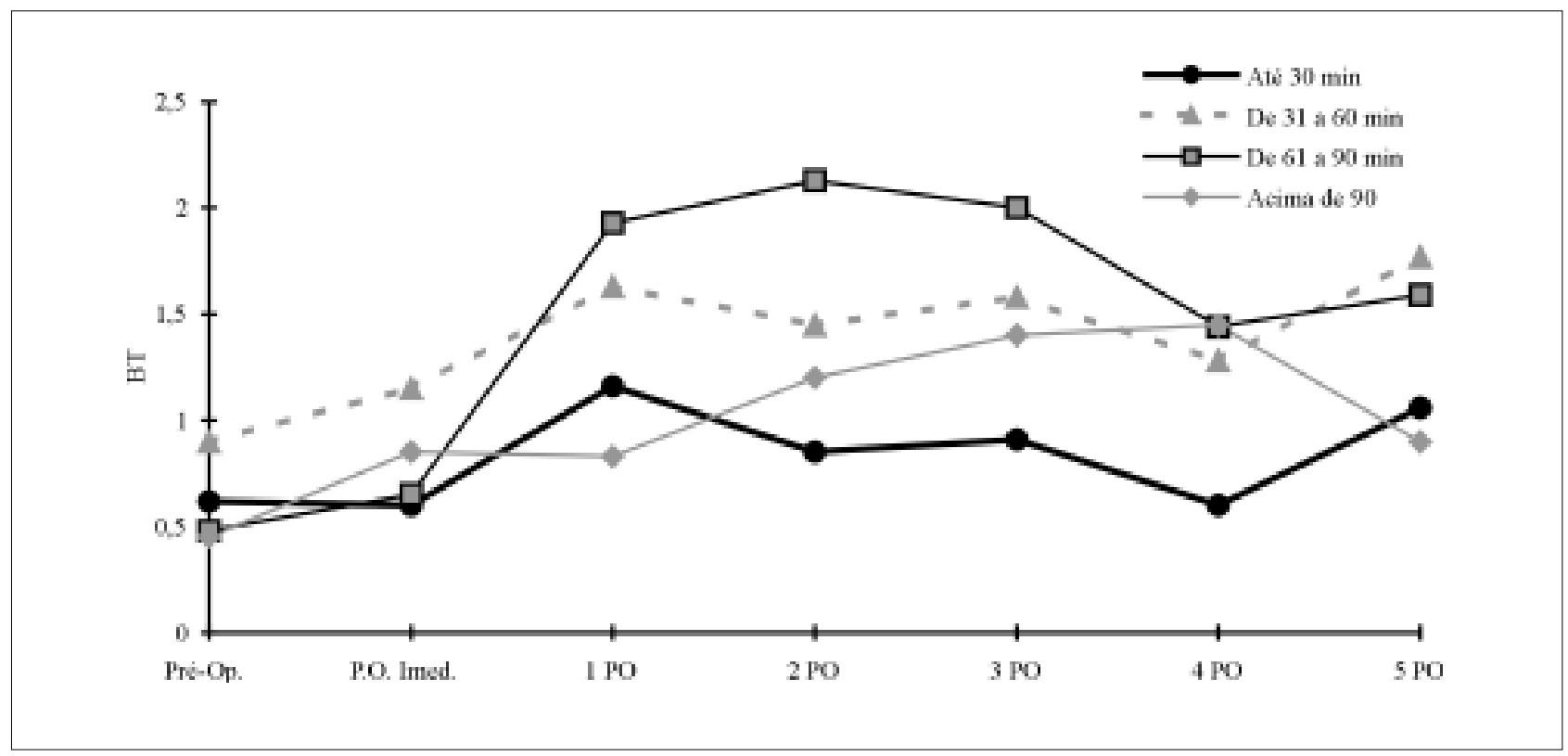

Figura 6 - Médias de bilirrubina total (BT) nos exames pré e pós-operatório, em relação ao tempo de clampeamento.

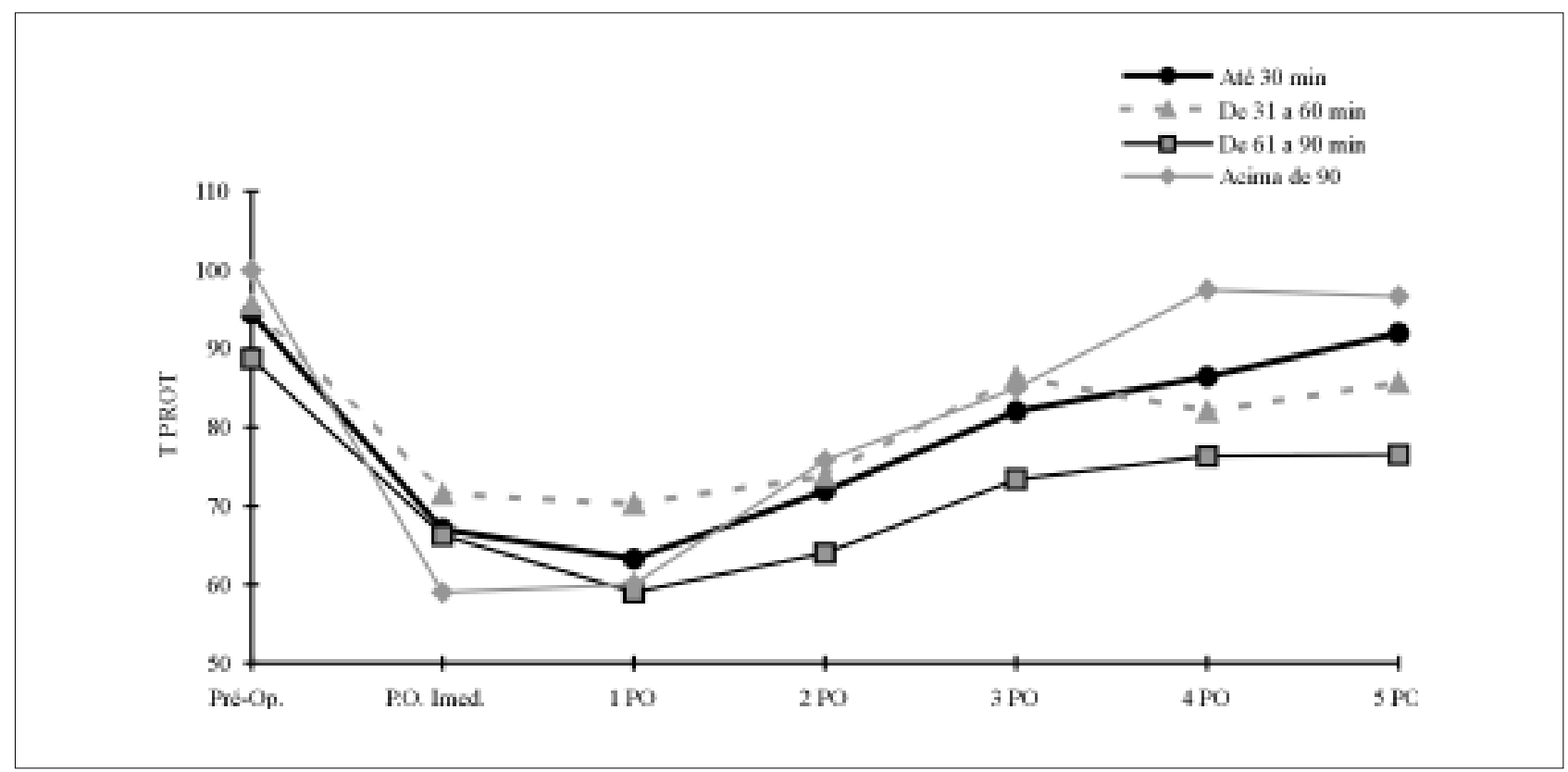

Figura 7 - Médias de tempo de protrombina $(T P)$ nos exames pré e pós-operatório, em relação ao tempo de clampeamento.

risco específicos do procedimento ${ }^{1}$, identificando aqueles potencialmente reversíveis ou tratáveis que deveriam ser corrigidos no período de preparo pré-operatório ${ }^{17}$. Em nosso estudo, avaliamos diversos fatores que poderiam influenciar a evolução dos pacientes submetidos à cirurgia hepática, bem como o efeito do clampeamento vascular aferente.

A mortalidade identificada, neste estudo $(6,7 \%)$, é bastante semelhante a outros relatos da literatura ${ }^{14,18,19}$, que apresentaram índices variando de $3,2 \%$ a $8,5 \%$ e até $9,7 \%$ para pacientes com neoplasia hepática maligna ${ }^{6,19}$. A per- da sangüínea perioperatória, apontada como um fator de risco prognóstico neste tipo de cirurgia ${ }^{20}$, não foi identificada como causa de mortalidade. Cabe ressaltar, no entanto, que o único caso que apresentou hemorragia intra-operatória maciça, definida como a necessidade de reposição de 10 ou mais unidades de sangue ${ }^{20}$, evoluiu para óbito.

A taxa de morbidade geral de $43,3 \%$, embora elevada, apresenta níveis plenamente aceitáveis, quando se considera a extensão e complexidade desse procedimento cirúrgico. Sitzmann e Greene, em um estudo semelhante, 
identificaram uma prevalência de complicações maiores de $33 \%$, sendo excluídas aquelas que não prolongaram a permanência hospitalar 5 .

A idade tem sido um fator controverso em alguns estudos. Yanaga et al. encontraram maior incidência de complicações infecciosas em pacientes com mais de 65 anos e mesmo quando comparadas as médias de idade, este foi um fator de risco significativo para processos infecciosos no período pós-operatório ${ }^{12}$. Na nossa análise, a idade foi identificada como fator de risco para morbidade, na análise univariada, quando comparada por faixas etárias e para pacientes com mais de 60 anos.

Iwatsuki e Starzl, ao relatarem sua experiência com hepatectomias, apresentaram alta taxa de doenças benignas $(44,3 \%)$, com mortalidade, significativamente menor, quando comparada às ressecções hepáticas por neoplasias malignas primárias de fígado ${ }^{19}$. $\mathrm{Na}$ análise isolada dos nossos dados, a cirurgia por doença maligna foi um fator de risco para morbidade, embora este achado não tenha sido confirmado por outros estudos ${ }^{1,7}$.

A taxa de complicações pós-operatórias tem sido relatada, com maior frequiência, em pacientes com hepatopatia crônica, mesmo naqueles sem descompensação clínica $\left(\right.$ Child A ${ }^{21}$, provavelmente, relacionadas com o processo isquêmico da oclusão vascular, embora o sangramento ainda seja um fator de risco mais grave do que a isquemia em pacientes cirróticos ${ }^{22}$. A colestase tem sido considerada um fator agravante mais importante que a cirrose, pois poderia traduzir algum grau de insuficiência hepática aguda com descompensação iminente ${ }^{22}$. Na série analisada neste estudo, a presença de parênquima hepático patológico foi um fator significativo na evolução dos dois grupos e a dosagem de bilirrubina total pré-operatória mostrou um nível de significância limítrofe quanto à morbidade.

A extensão da ressecção hepática, também, já foi considerada como fator de risco ${ }^{6}$, porém, atualmente, com o maior conhecimento da função de regeneração hepática e utilização dos processos de controle do fluxo sangüíneo ao órgão, estas ressecções estão se tornando progressivamente maiores sem aumento significativo dos índices de mortalidade ${ }^{23}$.

Huguet et al., ao apresentarem uma série de 50 pacientes submetidos a hepatectomias com oclusão vascular, não identificaram diferença quanto à mortalidade ou morbidade quando os grupos foram avaliados por tempo de isquemia, sugerindo que durante a cirurgia hepática devese tomar mais cuidado com a hemostasia do que com o tempo de clampeamento $^{4}$. Vários autores têm enfatizado a importância dos processos de oclusão vascular para redução do sangramento durante a cirurgia hepática ${ }^{2,10,20}$, no entanto, alguns serviços ainda utilizam apenas quando necessário, após o surgimento de hemorragia transoperató$\mathrm{ria}^{3}$, principalmente, pelo risco de lesões isquêmicas e pela diminuição do débito cardíaco durante o clampeamento $^{24}$.

Assim como a exclusão vascular completa, a oclusão vascular aferente total também permite a redução da hemorragia durante a secção do parênquima hepático, conforme já foi amplamente divulgado ${ }^{3}$. A isquemia hepática contínua e normotérmica apresenta conseqüências moderadas e transitórias sobre a função e nula sobre a regeneração hepática, mesmo quando se prolonga por períodos acima de 60 minutos, desde que o parênquima restante seja normal ${ }^{4,20}$. No caso de doença hepática crônica, o clampeamento pedicular intermitente ou a associação dele com a oclusão de veias hepáticas, por períodos de até 90 minutos, parece ser uma solução mais segura ${ }^{18,25}$. Por outro lado, os efeitos benéficos da isquemia intermitente, ou seja, a melhor tolerância do tecido hepático à isquemia prolongada e à descompressão do território esplâncnico, são contrabalançados pelos efeitos negativos do aumento de perdas sangüíneas e do processo de reperfusão do tecido pós-isquemia ${ }^{1}$. Na avaliação dos nossos dados, não foram identificadas diferenças na prevalência de morbidade com clampeamento contínuo ou intermitente. O tempo de clampeamento, também, não esteve relacionado com a morbidade ou mortalidade pós-operatória.

As perdas sanguíneas parecem ter uma relação direta com as complicações pós-operatórias, apresentando significância estatística para pacientes que necessitaram reposição de mais de uma unidade de sangue, nesta amostra. Gavelli et al. sugerem que as ressecções hepáticas podem ser realizadas com grande segurança, porém, a prevenção da hemorragia perioperatória deveria ser a preocupação essencial dos cirurgiões, considerando que se dispõe de várias alternativas entre os clampeamentos vasculares que permitem a intervenção em um órgão exangue ${ }^{1}$.

A variação do tempo de protrombina tem sido mais relacionado com a transfusão sanguínea volumosa do que com as ressecções extensas e, provavelmente, a presença de coagulopatia se deve, principalmente, à fibrinólise e seria menos influenciada pela deficiência de fatores de coagulação ${ }^{18}$. A função de síntese do parênquima hepático, avaliado pelo tempo de protrombina, após hepatectomias com oclusão vascular, apresenta uma piora acentuada no primeiro dia de pós-operatório e progressiva melhora ao longo da primeira semana, sendo que esta recuperação é mais lenta nos pacientes com hepatopatia crônica ${ }^{20,22}$. As dosagens de ALT e AST, no presente estudo, não apresentaram diferenças entre o grupo de pacientes com e sem complicações pós-operatórias. Quando os pacientes foram divididos, conforme o tempo de clampeamento pedicular, a dosagem no pós-operatório de bilirrubinas e do tempo de protrombina não foi diferente entre os diversos grupos, corroborando a hipótese de que não há relação entre o tempo de isquemia e a presença de complicações pós-operatórias. A variabilidade das enzimas hepáticas foi mais acentuada nos pacientes submetidos a um tempo de isquemia maior, porém, estes valores elevados retornaram aos níveis normais por volta da primeira semana.

Concluimos que a técnica de oclusão vascular aferente e o tempo de isquemia não apresentaram relação com a mortalidade ou morbidade pós-operatória nesta série de hepatectomias. As alterações das provas laboratoriais da função hepática retornam aos níveis pré-operatórios em aproximadamente uma semana. 


\begin{abstract}
Background: The control of blood loss during liver resection has been related to lower rates of perioperative mortality and morbidity. Techniques to minimize intraoperative bleeding are associated with blood flow interruption to the liver, either through an afferent vascular occlusion (Pringle maneuver) or by total vascular isolation of the organ. The aim of this study was to evaluate a series of partial hepatectomies with afferent blood flow occlusion, in patients with benign or malignant diseases. Method: Sixty hepatic resections with inflow occlusion, in 59 patients, were analyzed in order to search possible risk factors for morbidity and mortality, the connection between the hepatic ischemic time and the transaminases variation, the prothrombin time and bilirrubins and the postoperative evolution. Results: The prevalence of postoperative complications was $43 \%$ and the mortality rate was $6.7 \%$. The significant risk factor for mortality was the long lasting operative time when compared to patients who did not die. For the postoperative morbidity, the identified risk factors were age over sixty years old, surgery for malign neoplasm, abnormal liver parenchyma, blood loss demanding replacement of more than one unity of blood transfusion and another concomitant abdominal surgery. In a multiple regression analysis, those risk factors were reduced only to abnormal hepatic parenchyma. Conclusions: The ischemia time did not have any connection with the postoperative morbidity or mortality. The transaminases levels were higher in cases of longer ischemic time, however they returned to the preoperative levels in about a week. The transaminases variations were not different amongst patients who had postoperative morbidity or not.
\end{abstract}

Key Words: Liver; Ischemia; Morbidity; Inflow occlusion.

\section{REFERÊNCIAS}

1. Gavelli A, Ghiglione B, Huguet C. Facteurs de risque des hépatectomies: Résultats d'une étude multivariée - A propos de 113 cas. Ann Chir 1993; 47(7):586-591.

2. Pereira-Lima L. Hepatectomia parcial com impedimento total do fluxo sanguíneo aferente hepático. Rev AMRIGS 1993; 37(3):149-152.

3. Terblanche J, Krige JE, Bornman PC. Simplified hepatic resection with the use of prolonged vascular inflow occlusion. Arch Surg 1991; 126:298-301.

4. Huguet C, Gavelli A, Addario-Chieco P, et al. Liver ischemia for hepatic resection: Where is the limit? Surgery $1992 ; 11(3): 251-59$.

5. Sitzmann JV, Greene PS. Perioperative predictors of morbidity following hepatic resection for neoplasm - A multivariate analysis of a single surgeon experience with 105 patients. Ann Surg 1994; 219(1):13-17.

6. Savage AP, Malt RA. Elective and emergency hepatic resection: determinants of operative mortality and morbidity. Ann Surg 1991; 214(6):689-95.

7. Benoist $S$, Gailetton A, Gayral F. Intérêt du clampage sélectif dans les hépatectomies majeures. Ann Chir 1995; 49(7):589-95.

8. Kelly D, Emre S, Guy SR, et al. Resection of benign hepatic lesions with selective use of total vascular isolation. J Am Coll Surg 1996; 183:113-116.

9. Belli L, De Carlis L, Beati C, et al. Surgical treatment of symptomatic giant hemangiomas of the liver. Surg Gynecol Obstet 1992; 174(6):474-478.

10. Emre S, Schwartz ME, Katz E, et al. Liver resection under total vascular isolation - variations on a theme. Ann Surg 1993; 217(1):15-19.

11. Pringle JH. Notes on the arrest of hepatic hemorrhage due to trauma. Ann Surg 1908; 48:541-49.

12. Yanaga K, Kanematsu T, Sugimachi K, et al. Intraperitonial septic complications after hepatectomy. Ann Surg 1986; 203(2):148-152.

13. Couinaud C. Le foie. Etudes anatomiques et chirurgicales. Paris; Masson, 1957; 283-289.
14. Ribeiro J, Silveira VG, Pereira L, et al. Hepatectomias regradas: Análise de 135 casos. Rev CBC [tema livre 327]; 1995; 22 (supl 2):88.

15. Elias D, Lasser P, Debaene B, et al. Intermittent vascular exclusion of the liver (without vena cava clamping) during major hepatectomy. Br J Surg 1995; 82:1535-39.

16. Makuuchi M, Mori T, Guvén P, et al. Safety of hemihepatic vascular occlusion during resection of the liver. Surg Gynecol Obstet 1987; 164:155-58.

17. Torterolo E, Silva C, Cidade L, et al. Consideraciones técnico tácticas de la cirurgía hepática. Cir Uruguay 1994; 64:116-24.

18. Thompson HH, Tompkins RK, Longmire Jr WP. Major hepatic resection - A 25-year experience. Ann Surg 1983; 197(4):375-88.

19. Iwatsuki S, Starzl TE. Personal experience with 411 hepatic resections. Ann Surg 1988; 208(4):421-34.

20. Delva E, Camus Y, Nordlinger B, et al. Vascular occlusions for liver resections: operative management and tolerance to hepatic ischemia: 142 cases. Ann Surg 1989; 209(2):211-18.

21. Huguet C, Gavelli A, Bona S. Hepatic resection with ischemia of the liver exceeding one hour. J Am Coll Surg 1994; 178:454-58.

22. Emond J, Wachs M, Renz J, et al. Total vascular exclusion for major hepatectomy in patients with abnormal liver parenchyma. Arch Surg 1995; 130:824-831.

23. Houssin D, Massault PP, Pitre J, et al. Résection d'au moins six segments anatomiques en un temps pour tumeur du foie. Gastroenterol Clin Biol 1993; 17:750-57.

24. Delva E, Barberousse JP, Nordlinger B, et al. Hemodynamic and biochemical monitoring during major liver resection with use of hepatic vascular exclusion. Surgery 1984; 95:309-18.

25. Kim YI, Nakashima K, Tada I, et al. Prolonged normothermic ischaemia of human cirrhotic liver during hepatectomy: a preliminary report. Br J Surg 1993; 80:1566-70. 